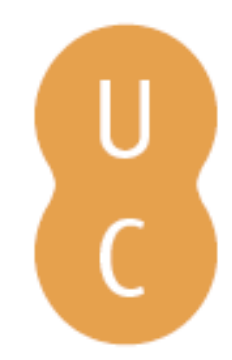

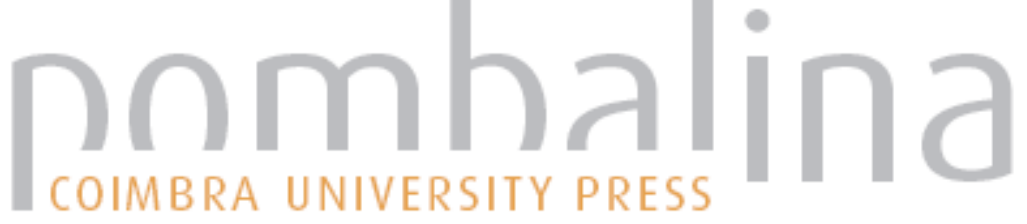

\section{Desemprego jovem e auto perceção de saúde mental}

Autor(es): $\quad$ Barros, Pedro Pita; Matias, Maria Ana; Moura, Ana

Publicado por: Imprensa da Universidade de Coimbra

URL

persistente:

URI:http://hdl.handle.net/10316.2/38283

DOI:

DOI:http://dx.doi.org/10.14195/978-989-26-1105-1_9

Accessed : $\quad$ 26-Apr-2023 00:57:11

A navegação consulta e descarregamento dos títulos inseridos nas Bibliotecas Digitais UC Digitalis, UC Pombalina e UC Impactum, pressupõem a aceitação plena e sem reservas dos Termos e Condições de Uso destas Bibliotecas Digitais, disponíveis em https://digitalis.uc.pt/pt-pt/termos.

Conforme exposto nos referidos Termos e Condições de Uso, o descarregamento de títulos de acesso restrito requer uma licença válida de autorização devendo o utilizador aceder ao(s) documento(s) a partir de um endereço de IP da instituição detentora da supramencionada licença.

Ao utilizador é apenas permitido o descarregamento para uso pessoal, pelo que o emprego do(s) título(s) descarregado(s) para outro fim, designadamente comercial, carece de autorização do respetivo autor ou editor da obra.

Na medida em que todas as obras da UC Digitalis se encontram protegidas pelo Código do Direito de Autor e Direitos Conexos e demais legislação aplicável, toda a cópia, parcial ou total, deste documento, nos casos em que é legalmente admitida, deverá conter ou fazer-se acompanhar por este aviso. 


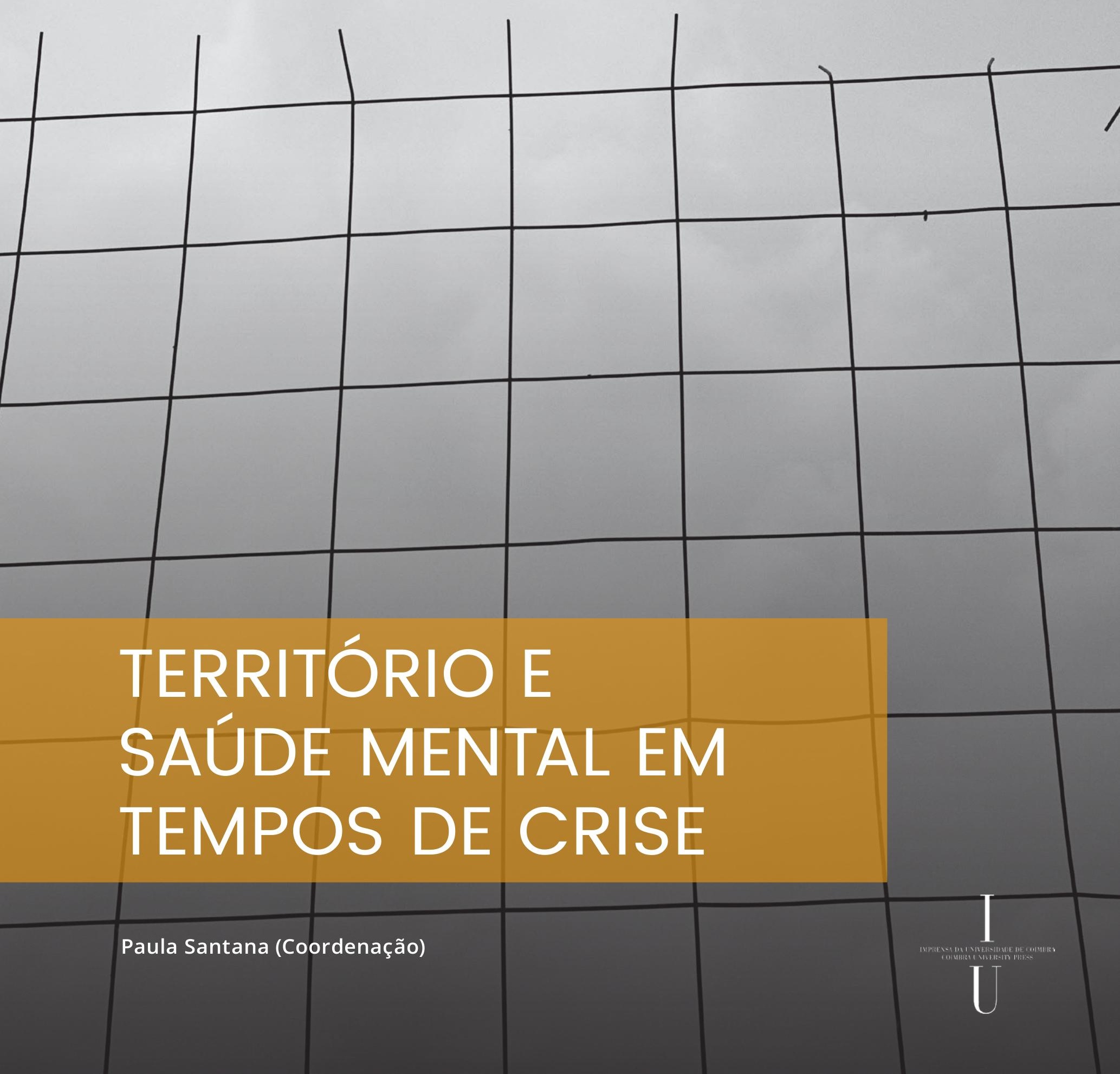




\subsection{Desemprego jovem e auto perceção de saúde mental}

Pedro Pita Barros ${ }^{(1,2)}$, Maria Ana Matias ${ }^{(1)}$ e Ana Moura ${ }^{(3)}$

(1) Nova School of Business and Economics, Universidade Nova de Lisboa; (2) Centre for Economic Policy Research (CEPR), Londres

(3) Universidade de Tilburg

DOI: http://dx.doi.org/10.14195/978-989-26-1105-1_9

Na recente crise económica, o desemprego jovem atingiu, em Portugal e na Europa, valores historicamente elevados. A taxa de desemprego entre os jovens atingiu os $34,8 \%$, o dobro do observado na população em geral. Uma questão natural é qual o efeito sobre a saúde mental nestes jovens, e saber se constituem um grupo a necessitar de atenção particular.

Utilizando o inquérito SMAILE, cobrindo cerca de 1500 indivíduos da área de Lisboa, foi calculada uma escala de saúde mental e vitalidade, recolhida da literatura científica. $O$ confronto desta escala com as características pessoais e de situação perante o emprego permitiu concluir que houve um impacto negativo relevante da crise económica (avaliada pela preocupação com as despesas diárias) sobre a saúde mental, que existe um gradiente positivo associado com anos de escolaridade (melhor saúde mental para quem possui mais anos de escolaridade) e uma grande importância do gradiente de escolaridade nos desempregados jovens (idade igual ou menor a 35 anos). Este gradiente específico é negativo: a saúde mental associada com o desemprego jovem tem menor valor na escala usada quanto maior for o nível de escolaridade. Em termos quantitativos, este efeito é substancialmente mais elevado que qualquer um dos outros efeitos mencionados. A vulnerabilidade encontrada deverá motivar políticas conjuntas de promoção do emprego e da saúde mental neste grupo etário.
During the Great Recession, youth unemployment reached, in Portugal and in Europe, historically high values. The youth unemployment rate in Portugal reached $34,8 \%$, twice the value of the general population. A natural question is the impact on the mental health of the young of this high unemployment rate, and ask whether, or not, this is a vulnerable group requiring specific attention from public policies.

Taking advantage of the SMAILE survey, which covers about 1500 individuals in the Lisbon region, we compute a mental health and vitality scale that is present in the literature. Relating this scale with personal characteristics and with the employment situation suggests a negative impact on mental health from the economic crisis (measured at the individual level by the concern with daily expenditures). We also find a positive gradient associated with schooling (better mental health for those that had more years of schooling completed) and a more important gradient of schooling in the young unemployed (defined as unemployed aged equal or less than 35 years). Moreover, this specific gradient is negative: mental health associated with youth unemployment is lower the higher the level of schooling. In quantitative ter$\mathrm{ms}$, this effect is substantially larger than any of the other effects found. This mental health vulnerability of young unemployed should motivate joint policies addressing unemployment and mental health specifically designed for this age group. 


\section{Introdução}

A Organização Mundial de Saúde (OMS) define a saúde como o "estado de completo bem-estar físico, mental e social e não somente ausência de afecções e enfermidades"(World Health Organization [WHO], 2007). A saúde mental desempenha pois um papel crucial na saúde dos indivíduos.

Ao longo do tempo as sociedades têm vindo, de uma forma crescente, a tomar consciência da importância da saúde mental. Vários estudos comprovam que uma sociedade com uma elevada taxa de incidência de doenças do foro mental resulta em perda de produtividade, a qual se traduz em elevados custos económicos (OECD, 2014). Desta forma, promover uma boa saúde mental dos indivíduos tem sido uma das prioridades das atuais sociedades uma vez que se traduz em ganhos de produtividade e prosperidade, crucial ao crescimento económico (WHO, 2011).

São vários os fatores de risco/agravamento com impacto negativo na saúde mental da população. Entre esses fatores encontram-se as características individuais e familiares, o contexto escolar, fatores relacionados com a comunidade e culturais bem como acontecimentos de maior stresse que podem ocorrer ao longo da vida, dos quais se destaca a situação de desemprego (OECD, 2012).

A questão do desemprego tem sido amplamente discutida devido às suas repercussões tanto ao nível individual como coletivo. O desemprego está associado, entre outros fatores, a perda de rendimento, instabilidade financeira, estigma social, perda de autoestima e diminuição da atividade física e mental do indivíduo (Artazcoz et al., 2004; OECD 2012). Todos estes fatores tem um impacto negativo na saúde mental uma vez que contribuem para um aumento (ou agravamento) das doenças associadas à depressão e ansiedade (WHO, 2011).. Adicionalmente, os jovens desempregados têm um maior risco de contrair problemas de saúde mental quando comparados com os jovens que permanecem empregados (WHO, 2011).
Desta forma os desempregados, sobretudo os jovens desempregados, são considerados como um grupo vulnerável aos quais estão associados níveis de saúde mental mais baixos quando comparados com os da generalidade da população (Artazcoz et al., 2004).

Devido à recente crise económica e financeira mundial, à qual está associada um aumento significativo da taxa de desemprego, nomeadamente da taxa de desemprego jovem ${ }^{1}$, surgiu uma maior preocupação sobre os seus efeitos na saúde mental dos indivíduos.

De acordo com o recente relatório da Organização Mundial do Trabalho de 2015, a taxa global de desemprego jovem teve um crescimento acentuado entre 2007 e 2010 de $11,7 \%$ para $13 \%$, respetivamente. Entre os anos de 2012 e 2014, esta taxa estagnou permanecendo nos 13\%. É estimado que em 2015 essa taxa aumente ligeiramente para 13,1\% (ILO, 2015).

Em 2014, a taxa de desemprego jovem na zona Euro atingiu os $23 \%$. No entanto, e apesar de uma melhoria na taxa de desemprego entre 2012 e 2014, a taxa de desemprego jovem em 2014 continua a exceder os $30 \%$ em vários países da Europa, nomeadamente, Grécia (52,4\%), Itália $(42,7 \%)$, Portugal $(34,8 \%)$ e Espanha $(53,2 \%)$ (ILO, 2015), valores muito acima da taxa de desemprego geral de cada um destes países.

Adicionalmente, o problema do desemprego jovem ainda se torna mais grave quando olhamos para a sua duração. Na União Europeia (28 países) a taxa de jovens desempregados à procura de emprego há mais de um ano aumentou de $32,6 \%$ para $35,5 \%$ entre 2012 e 2014, respetivamente (ILO, 2015).

Desta forma, e sendo Portugal um dos países com pior performance ao nível da taxa de desemprego jovem, tem o enquadramento ideal para determinar o impacto do desemprego jovem na saúde mental. 
Com o objetivo de determinar o impacto do desemprego jovem na saúde mental, usou-se informação de um inquérito realizado entre 2014 e 2015 nos municípios da Amadora, Lisboa, Mafra e Oeiras. Desta forma avalia-se o impacto do desemprego jovem na perceção da sua saúde mental. O nosso contributo consiste na análise detalhada deste impacto recorrendo a estatística descritiva, nomeadamente com o recurso a histogramas e funções densidade. Desta forma, e ao contrário das análises econométricas que se centram na média ${ }^{2}$ , esta metodologia permite a avaliação de dinâmicas que ocorrem ao longo de toda a distribuição e não nos focamos apenas nas dinâmicas que ocorrem em torno da média.

Este estudo está integrado no projeto SMAILE (Saúde Mental - Avaliação do Impacto das condicionantes Locais e Económicas) que visa avaliar o efeito ambiental e territorial das determinantes em saúde na saúde mental e o uso dos serviços de saúde mental em tempos de crise económica e social.

O estudo está dividido da seguinte forma: a secção 2 descreve a fonte dos dados, a secção 3 procede à análise estatística dos dados e a secção 4 apresenta as principais conclusões.

\section{Dados}

Os dados utilizados nesta análise foram obtidos através de um inquérito realizado entre os meses de Agosto de 2014 e Fevereiro de 2015. Este inquérito foi feito tanto presencialmente como online e destinou-se a toda a população adulta (maiores de 18 anos) residente nos municípios da Amadora, Lisboa, Mafra e Oeiras. A escolha destes quatro concelhos da Área Metropolitana de Lisboa foi feita com base no seu grau de desenvolvimento com o objetivo de termos na nossa amostra áreas urbanas consolidadas (Lisboa), áreas urbanas recentes e em crescimento (Amadora e Oeiras) e áreas menos desenvolvidas (Mafra).
A população estatística consiste em 808.110 residentes, na qual foi recolhida uma amostra aleatória simples de 1.609 residentes (estimouse uma margem de erro de $3,21 \%$ a um nível de confiança de $99 \%$ ).

O inquérito recolheu informação individual ao nível das características demográficas (idade e género); das características socioeconómicas (nível de educação, status profissional, estado civil, situação financeira do agregado familiar e preocupações financeiras); da saúde (diabetes, hipertensão, índice de massa corporal); das características comportamentais (fumador, atividade física) e da auto perceção da saúde mental.

Relativamente à auto perceção da saúde mental, esta foi avaliada com base nas escalas de saúde mental e vitalidade (MHVS), versão validada para a população Portuguesa do Short Form 36-item Health (SF-36v2). O SF-36 é um questionário de estado de saúde com o objetivo de medir e avaliar o estado de saúde de populações e indivíduos com ou sem doença; monitorizar doentes com múltiplas condições, comparar doentes com condições diversas e comparar o estado de saúde de doentes com o da população em geral. O questionário contém 36 itens cobrindo oito dimensões de estado de saúde e detetando tanto os estados positivos como os negativos de saúde (Ferreira, 2000).

O MHVS é medido numa escala de 0 a 100 , em que o valor máximo corresponde à melhor situação de saúde mental possível. Este indicador foi calculado seguindo a metodologia proposta por Ware e outros (1993). Devido à falta de um grupo de controlo, foi estipulado um valor de corte de 50 (ponto médio da escala) para determinar os indivíduos que estão melhor e os que estão pior em termos de saúde mental medida pela escala. De salientar que o inquérito recolheu outro tipo de informação, nomeadamente ao nível do meio envolvente onde o indivíduo reside, mas essa informação não é considerada no presente estudo, sendo tratada em detalhe por outros autores. 


\section{Análise descritiva}

A secção apresenta dados estatísticos sobre a amostra usada para o presente trabalho, focando sobretudo na escala de saúde mental e vitalidade (MHVS). A análise relaciona a componente da perceção da saúde mental com a idade (faixa etária), género, nível de escolaridade e condição perante o mercado de trabalho (secção 3.1).

Dado que a questão relevante é qual o impacto do desemprego jovem na perceção da saúde mental, a secção 3.2 dedica-se a uma análise estatística considerando os indivíduos jovens e desempregados, a qual assenta nas mesmas variáveis usadas na secção 3.1.

Finalmente, e apesar de não haver representatividade ao nível do concelho, na secção 3.3 procedemos a uma análise da perceção da saúde mental a esse nível.

\subsection{Perceção da saúde mental e suas determinantes}

Em primeiro lugar, utilizando os dados recolhidos no inquérito anteriormente descrito, é analisada a distribuição da escala de saúde mental e vitalidade correspondente à auto avaliação dos indivíduos. Através da figura 1, que apresenta o respetivo histograma e função densidade, verifica-se que a distribuição segue aproximadamente uma distribuição normal, ligeiramente enviesada à esquerda. A grande maioria dos indivíduos tem uma pontuação que se encontra entre os $40 \mathrm{e}$ os 85 , sendo que a massa concentrada nos extremos da distribuição é reduzida. Desta forma, e uma vez que a grande maioria dos indivíduos apresenta uma pontuação acima dos 50 , podemos afirmar que a perceção dos indivíduos sobre a sua saúde mental é boa.

Em termos de indicadores que ajudem a caracterizar a distribuição da escala de saúde mental e vitalidade de forma mais concreta, tem-se a média e a mediana, que tomam valores de 58 e 61, respetivamente. $\mathrm{O}$ desvio padrão da distribuição é de 19,5. Em termos de percentis da distribuição, estes são apresentados no quadro 1.

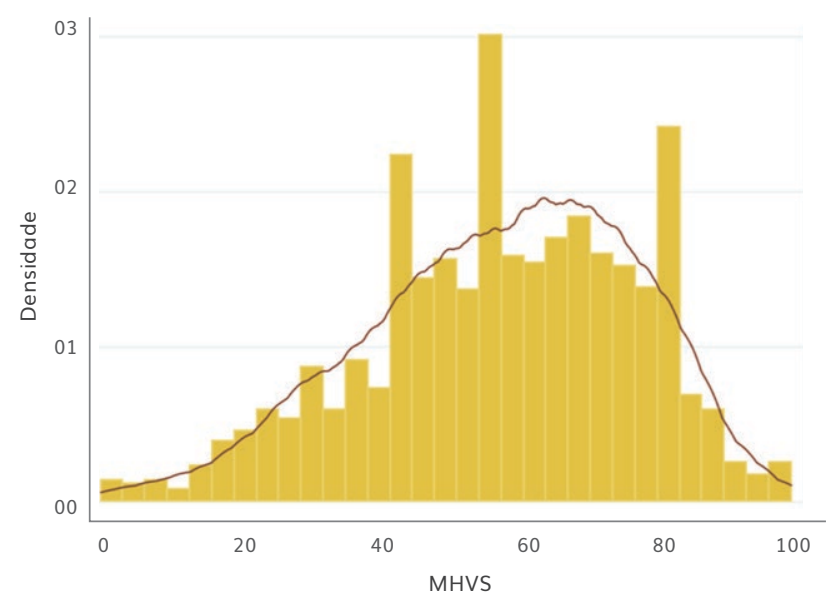

Figura 1. Histograma e função densidade MHVS

Fonte: Fonte: elaboração própria a partir dos questionários realizados nos municípios da Amadora, Lisboa, Mafra e Oeiras, em 2014 e 2015

Quadro 1. Percentis da distribuição do MHVS

\begin{tabular}{|c|c|}
\hline \multicolumn{1}{|c|}{ Percentil } & Valor MHVS \\
\hline 5 & 22 \\
\hline 25 & 44 \\
\hline 50 (mediana) & 61 \\
\hline 75 & 72 \\
\hline 95 & 86 \\
\hline
\end{tabular}

Fonte: elaboração própria a partir dos questionários realizados nos municípios da Amadora, Lisboa, Mafra e Oeiras, em 2014 e 2015

Dado que se assume que o valor de 50 na escala MHVS é o limiar entre um estado de saúde positivo ou negativo, é possível verificar que, mais de $50 \%$ dos indivíduos inquiridos tem uma saúde mental acima deste limiar (quadro 1). Importa, então, avaliar que características estão associadas positiva e negativamente com a MHVS.

A idade é tida na literatura como sendo um dos principais determinantes da saúde mental dos indivíduos. Ao contrário de outras doenças, as doenças do foro psiquiátrico, nomeadamente as doenças mentais graves, são mais prevalentes no final da adolescência/início da idade adulta e com novo pico por volta dos 30 anos de idade. De acordo com o último Estudo Epidemiológico (Caldas de Almeida \& Xavier, 2013), a faixa etária entre os 18 e os 24 anos tem maior probabilidade de desenvolver uma doença mental comparativamente com as restantes faixas etárias. De salientar, que os jovens têm uma maior prevalência $(50,1 \%)$ de desenvolver pelo menos uma doença mental (Caldas de Almeida \& Xavier, 2013). 
Ao analisar a relação entre a idade e a escala de saúde mental e vitalidade na amostra utilizada (figura 2), não parece existir uma relação clara entre as duas variáveis. O coeficiente de correlação de $-0,181$ evidência um grau de correlação fraco (Dancey \& Reidy, 2004), dando suporte a esta conclusão.

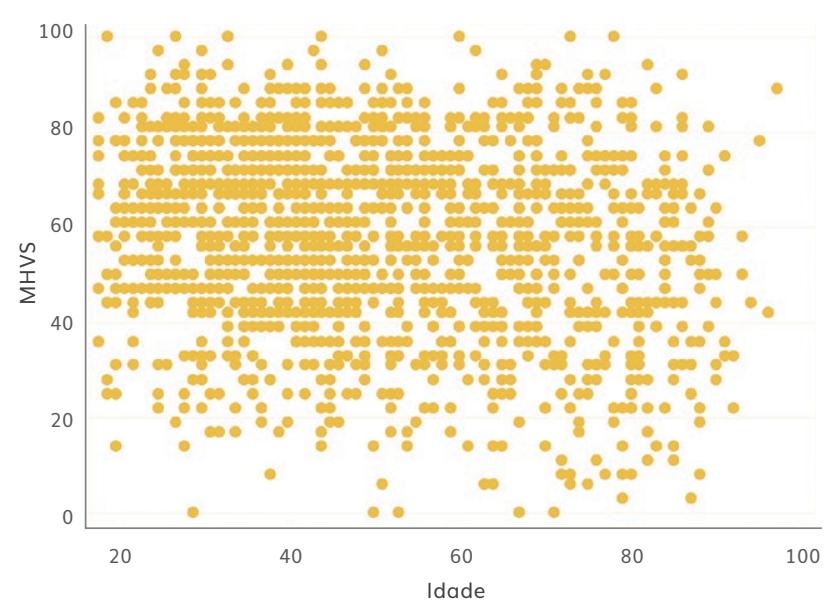

Figura 2. Relação entre idade e MHVS

Fonte: elaboração própria a partir dos questionários realizados nos municípios da Amadora, Lisboa, Mafra e Oeiras, em 2014 e 2015

De forma a avaliar a existência de eventuais diferenças no que toca à distribuição da MHVS por faixas etárias, os indivíduos que compõem a amostra são divididos por quatro faixas etárias: indivíduos com idade igual ou inferior a 35 anos; indivíduos com idades compreendidas entre os 36 e os 50 anos; indivíduos entre os 51 e os 65 anos; e, finalmente, indivíduos com idade superior a 65 anos. A figura 3 ilustra as funções densidade da distribuição da escala da saúde mental auto reportada para cada uma das faixas etárias consideradas.

O valor médio de MHVS é de 61,9 na distribuição correspondente à faixa etária mais jovem e vai diminuindo sucessivamente para 59,2 e 57,4 nas duas faixas etárias seguintes. Entre indivíduos pertencentes ao escalão etário superior, este valor é de 53,3. Portanto pode-se afirmar que à medida que se avança para faixas etárias mais elevadas, o valor médio da escala de saúde mental auto reportada pelos indivíduos decresce, o que não é facilmente percetível apenas com a análise gráfica (figura 3).

Simultaneamente, verifica-se um aumento progressivo da dispersão da distribuição. Isto é, enquanto na faixa etária abaixo dos 35 anos a massa da distribuição se encontra relativamente concentrada em redor do pico, esse padrão vai-se alterando à medida que se passa para as faixas etárias seguintes. O escalão etário composto por indivíduos com idade superior a 65 anos exibe já uma dispersão de valores muito superior, com maior massa nos extremos da distribuição. Os valores da variância, que medem o grau de dispersão de uma distribuição, ilustram esta situação: a variância toma valores de 317,1 na distribuição da MHVS no escalão etário inferior, aumentando progressivamente até atingir os 457,4 no escalão etário superior.
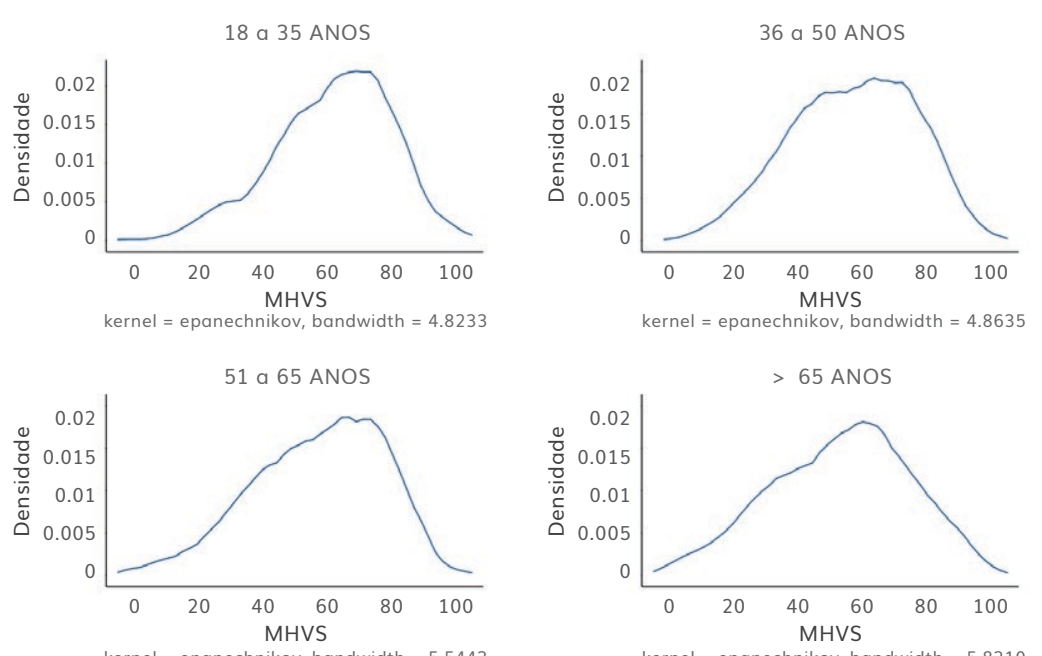

Figura 3. Funções densidade da distribuição MHVS

Fonte: elaboração própria a partir dos questionários realizados nos municípios da Amadora, Lisboa, Mafra e Oeiras, em 2014 e 2015
Assim, apesar de com base na figura 2 não ser possível identificar uma relação clara entre a idade e o estado de saúde mental auto reportado pelos indivíduos, a verdade é que, atentando às distribuições da MHVS por escalão etário, parece existir evidência de uma deterioração da saúde mental com a idade. Apesar das doenças mentais serem mais prevalentes nos jovens, esta relação negativa entre idade e saúde mental pode ser explicada com base no facto de indivíduos mais velhos enfrentarem situações de mais stress como por exemplo a passagem à reforma, 
a qual está associada com um decréscimo do status socioeconómico. Estes fatores estão associados ao isolamento, perda de independência, solidão e sofrimento psicológico (OECD, 2015).

Para além da idade, também o género é um determinante da saúde mental dos indivíduos. Tem-se observado que as mulheres tendem a reportar um pior estado de saúde do que os homens. Tal também sucede nos nossos dados, assim como ilustra a figura 4, onde são sobrepostas as funções densidade dos homens e das mulheres. De acordo com a figura 4, a distribuição da escala de saúde mental auto reportada
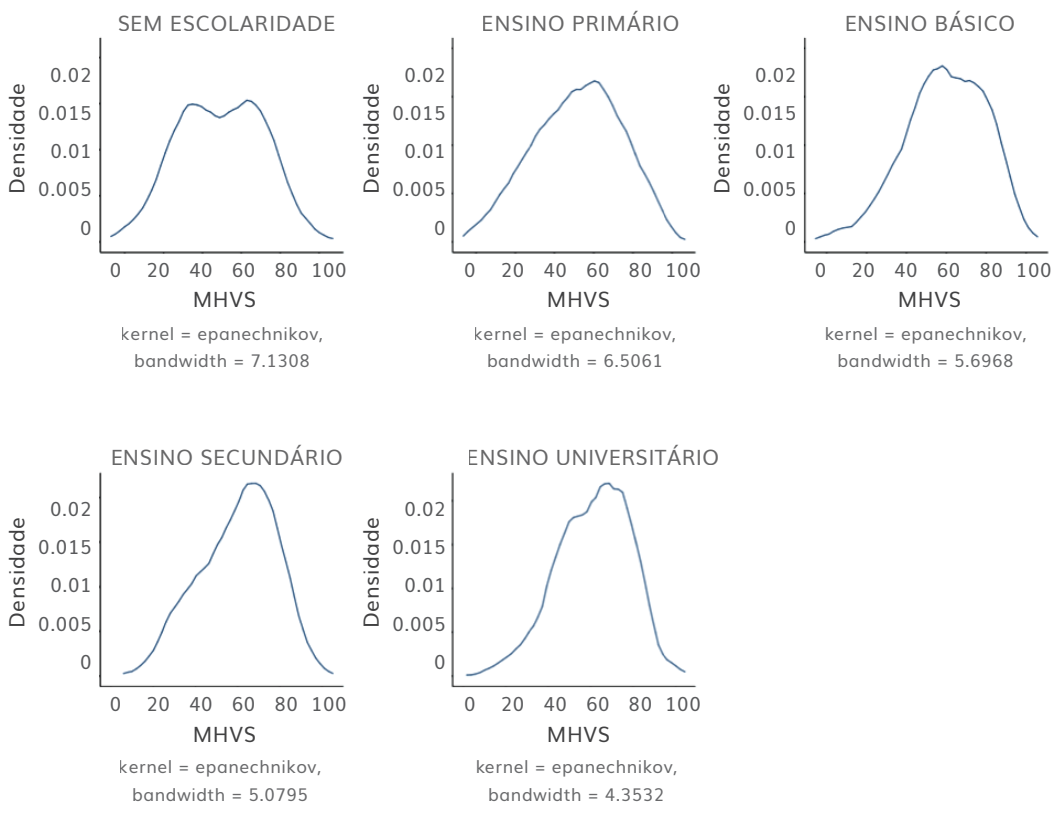

Figura 5. Distribuição MHVS por nível de escolaridade Fonte: elaboração própria a partir dos questionários realizados nos municípios da Amadora, Lisboa, Mafra e Oeiras, em 2014 e 2015 dos homens está localizada à direita da das muIheres. O valor médio da distribuição é de 63,6 e 54,8 para os homens e mulheres, respetivamente. Adicionalmente, a distribuição da MHVS entre as mulheres exibe um grau de achatamento superior ao dos homens. Por outras palavras, a distribuição da MHVS entre os homens exibe um pico mais acentuado do que a das mulheres.

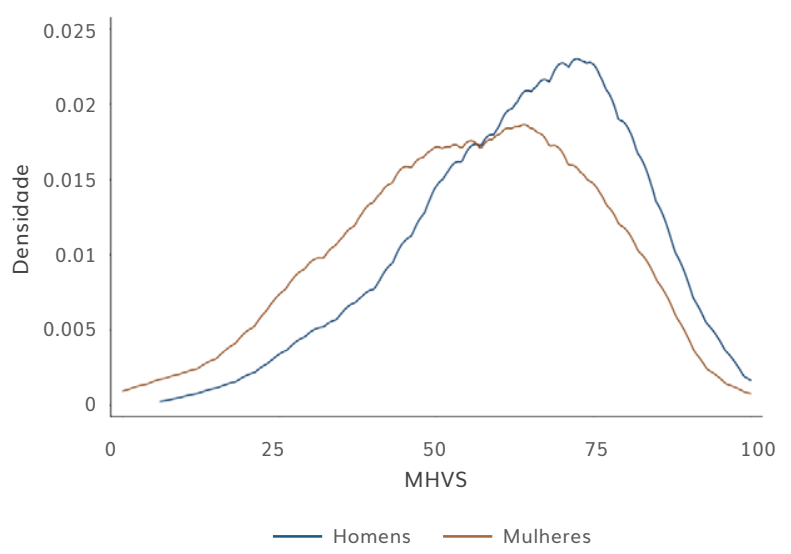

Figura 4. Distribuição MHVS por género

Fonte: elaboração própria a partir dos questionários realizados nos municípios da Amadora, Lisboa, Mafra e Oeiras, em 2014 e 2015

Outra das variáveis frequentemente relacionadas com o estado de saúde mental é o nível educacional dos indivíduos. De acordo com a literatura, indivíduos com maior nível de escolaridade têm um melhor estado de saúde mental (OECD,
2012), . A figura 5 apresenta a distribuição da escala de saúde mental auto reportada por nível de escolaridade. Mais uma vez, são evidentes as alterações que ocorrem na distribuição à medida que se avança no nível de escolaridade dos indivíduos. Não só a média da distribuição vai aumentando gradualmente com o nível de educação - passando de 49,5 entre indivíduos sem escolaridade para 61,2 entre aqueles que possuem estudos universitários - como o grau de dispersão também vai diminuindo.

Entre os determinantes do estado de saúde mental, encontra-se também a situação profissional dos indivíduos, sendo que esta variável assume uma importância considerável. De facto, ter uma ocupação profissional não é apenas uma forma de obter rendimento para o consumo de bens e serviços, mas é também uma forma de os indivíduos se sentirem úteis e realizados, alargarem o seu círculo de relacionamentos ou alcançarem determinada posição na sociedade. Adicionalmente, possuir uma ocupação profissional facilita aos indivíduos a organização do seu dia-a-dia e a gestão do tempo. Desta forma, uma situação de desemprego é frequentemente motivo de grande tensão, podendo mesmo ser responsável pelo desenvolvimento de depressões ou outras doenças do foro mental (Artazcoz et al., 2004; OECD, 2012; WHO, 2011). 
A figura 6 apresenta a função densidade da escala de saúde mental e vitalidade entre indivíduos empregados e desempregados. Indivíduos com outro tipo de situação profissional, tais como estudantes ou domésticos, não foram considerados na construção desta figura.

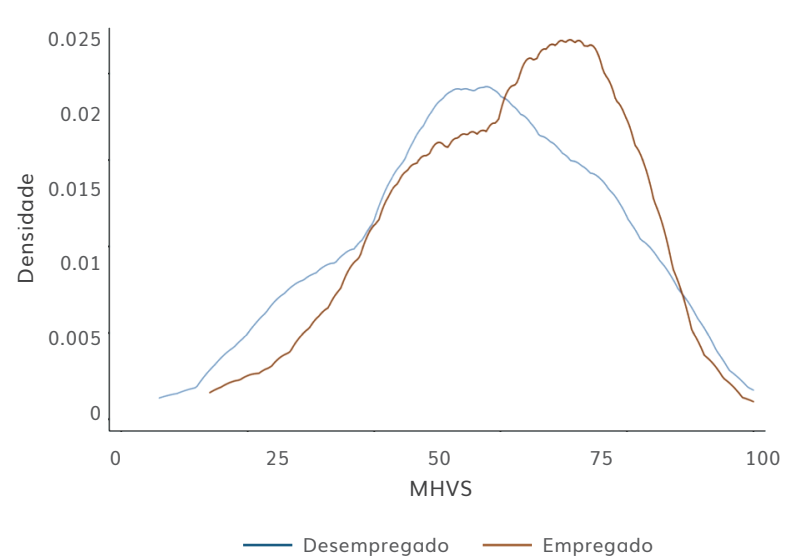

Figura 6. Distribuição MHVS entre indivíduos empregados/desempregados

Fonte: elaboração própria a partir dos questionários realizados nos municípios da Amadora, Lisboa, Mafra e Oeiras, em 2014 e 2015

À semelhança do que se verificou em relação às diferenças de género, também a função densidade entre indivíduos desempregados se situa à direita da daqueles que estão empregados. Os valores médios são de 56,6 e 61,1, respetivamente, entre indivíduos desempregados e empregados.

Existem algumas teorias na literatura que procuram explicar que grupos de indivíduos seriam mais afetados, em termos do estado de saúde mental, por uma situação de desemprego. Resumidamente, um conjunto de teorias sugere que os indivíduos mais afetados por uma situação de desemprego são os mais privilegiados em termos de tipo de atividade e salário, pois são estes que sofrem a maior quebra no seu nível de vida (Dukerich, 2001). Por oposição, há teorias que apontam para o facto de serem aqueles com menores recursos socioeconómicos os que mais sofrem com uma situação de desemprego. O racional por detrás desta teoria prende-se com o facto de estar à partida inserido num contexto desvantajoso em termos socioeconómicos atuar como um fator que vem exacerbar o efeito negativo que uma situação de desemprego exerce na saúde mental dos indivíduos (Syme, 1996).

\subsection{Desemprego jovem e perceção da saúde mental}

Deste ponto em diante, será dado especial enfoque ao desemprego como variável passível de afetar a perceção da saúde mental. Sem qualquer ambição de estabelecer relações de causalidade, dadas as características da amostra recolhida, entre variáveis socioeconómicas e o efeito de uma situação de desemprego no estado de saúde mental auto reportado, serão analisados os histogramas e funções densidade da escala de saúde mental e vitalidade de acordo com algumas interações das variáveis socioeconómicas anteriormente mencionadas. O objetivo é apenas avaliar o comportamento da distribuição entre grupos distintos de indivíduos.

É frequentemente encontrada evidência na literatura que aponta no sentido de serem os indivíduos do género masculino aqueles a quem uma situação de desemprego produz efeitos mais nefastos no estado de saúde mental. Esta situação deve-se essencialmente ao papel de sustento tradicionalmente atribuído ao homem no seio do agregado familiar, sendo que, na impossibilidade de manter esse papel, o homem experimenta uma sensação de falhanço pessoal (Paul \& Moser, 2009; Backhans \& Hemmingson, 2012; Breslin \& Breslin, 2013; Clemens et al., 2015). As mulheres, por outro lado, não só não são vistas como as principais responsáveis pelo sustento do agregado familiar, como também têm maior facilidade em substituir o seu papel de profissionais pelo de cuidadora do lar (Artazcoz et al., 2004).

Através da análise da figura 7, verifica-se que a função densidade entre as mulheres está localizada ligeiramente à direita da dos homens. No entanto, esta situação não é tão notória como quando se considera a totalidade da amostra (figura 4). $\mathrm{Na}$ verdade, as maiores diferenças de género no que toca à função densidade da escala de saúde mental e vitalidade ocorrem quando se considera apenas os indivíduos empregados, como ilustrado na figura 8. Estes resultados podem estar associados à insatisfação laboral, sugerindo que a importância assumida pelo tipo de trabalho desempenhado é fundamental para a manutenção de um bom estado de saúde mental. 


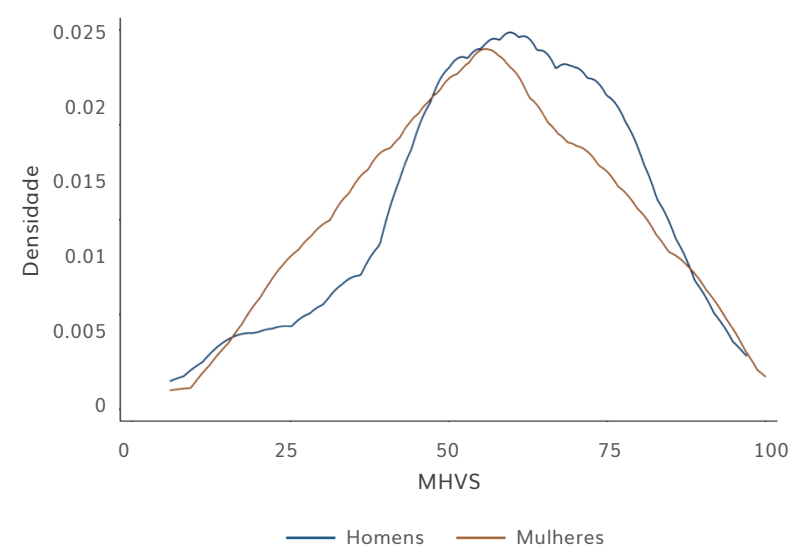

Figura 7. Distribuição MHVS por género e indíviduos desempregados Fonte: elaboração própria a partir dos questionários realizados nos municípios da Amadora, Lisboa, Mafra e Oeiras, em 2014 e 2015

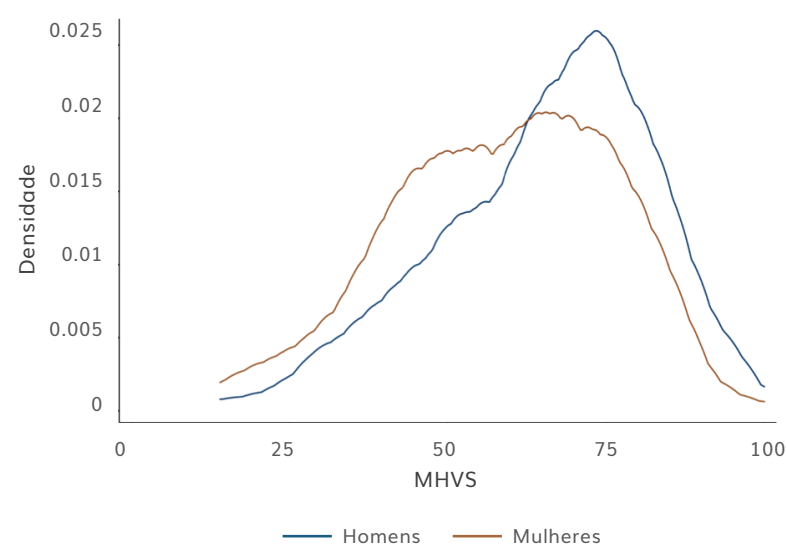

Figura 8. Distribuição MHVS por género e indivíduos empregados Fonte: elaboração própria a partir dos questionários realizados nos municípios da Amadora, Lisboa, Mafra e Oeiras, em 2014 e 2015

Dentro da temática geral do desemprego, é o desemprego jovem que tem gerado mais preocupações. Efetivamente, e como mencionado anteriormente, a taxa de desemprego entre os jovens na União Europeia é o dobro da dos adultos. Sendo a entrada no mercado laboral após término da educação formal uma fase crucial para delinear uma carreira profissional, uma situação de desemprego pode colocar em risco o futuro dos indivíduos, principalmente se for de longa duração. De forma a combater esta questão foi desenvolvida uma estratégia a nível europeu de combate ao desemprego jovem. Esta consiste num pacote de medidas que incluem, por exemplo, a melhoria da oferta e qualidade dos estágios empresariais para os jovens (Banerji et al., 2014; European Commission, 2015).

Apesar de, quando se considera toda a amostra, os jovens serem aqueles com melhor estado de saúde mental auto reportada (figura 3), se aten- tarmos apenas ao grupo de indivíduos desempregados, tal já não se verifica. De facto, de acordo com a figura 9, a função densidade da escala de saúde mental e vitalidade parece deslocar-se para a direita à medida que se avança para escalões etários superiores. Indicadores como a média ou o desvio padrão não transmitem esta realidade, dado que se mantêm relativamente constantes para os três grupos etários considerados. No entanto, a figura 9 ilustra claramente as alterações que ocorrem a nível da forma da distribuição.

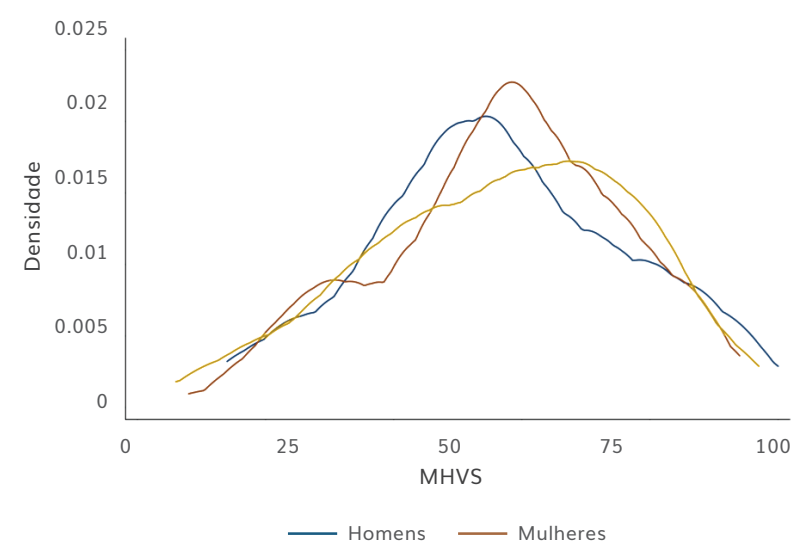

Figura 9. Distribuição MHVS por faixa etária

Fonte: elaboração própria a partir dos questionários realizados nos municípios da Amadora, Lisboa, Mafra e Oeiras, em 2014 e 2015

Realizando uma análise semelhante à da figura anterior, mas tendo em conta o nível de escolaridade dos indivíduos ao invés do escaIão etário obtêm-se as funções densidade para indivíduos que se encontram em situação de desemprego, por nível educacional. Estas são apresentadas na figura 10.

Apesar de não muito aparente através da simples observação das funções densidade exibidas na figura 10, a análise dos valores médios da distribuição revela um padrão interessante: ao considerarmos a totalidade de indivíduos na amostra obtínhamos uma relação positiva entre o valor médio da escala de saúde mental e vitalidade e o nível educacional dos indivíduos (figura 5); no entanto, ao restringir a análise a indivíduos que se encontram em situação de desemprego, tal deixa de se verificar. Na verdade, o valor médio da MHVS mais elevado ocorre entre aqueles que estão desempregados e não possuem qualquer tipo de escolaridade $(61,3)$. Aque- 

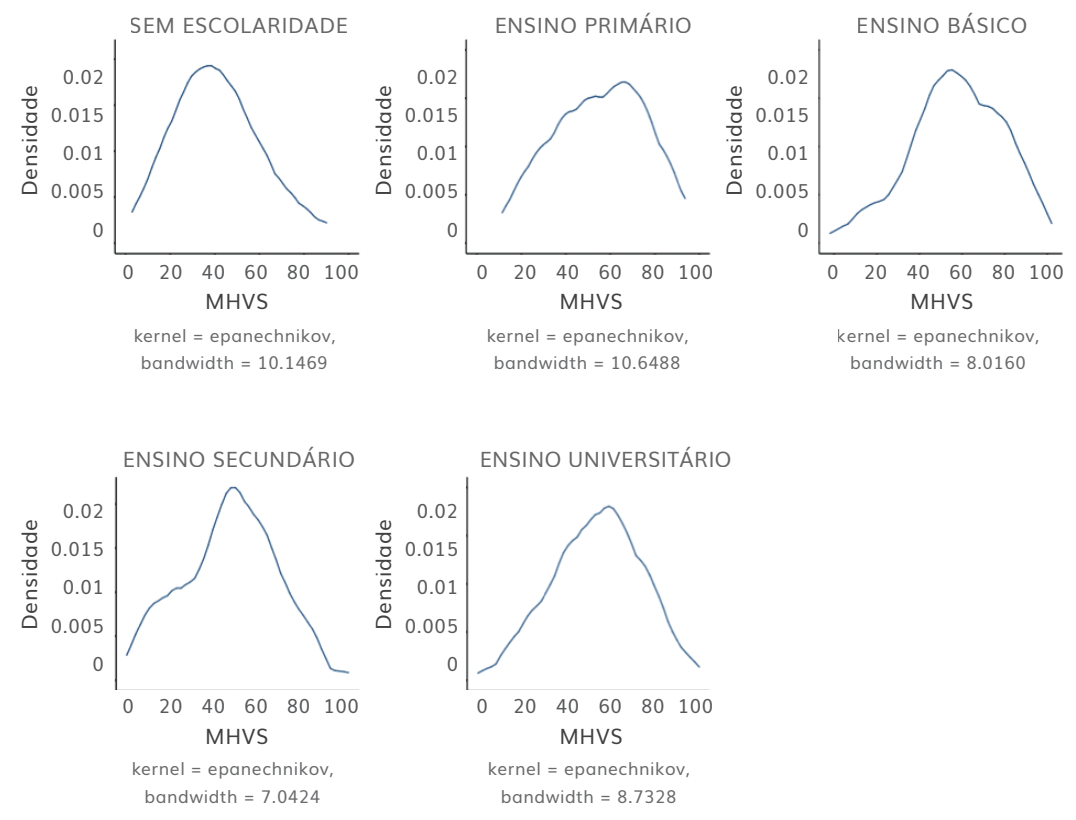

Figura 10. Distribuição MHVS por nível de escolaridade Fonte: elaboração própria a partir dos questionários realizados nos municípios da Amadora, Lisboa, Mafra e Oeiras, em 2014 e 2015

les que completaram o ensino universitário e se encontram numa situação de desemprego, pelo contrário, são o grupo com menor valor médio da MHVS $(54,1)$. Nos restantes grupos o valor médio da escala de saúde mental e vitalidade é de 55,3, 57,4 e 56,3, respetivamente para indivíduos que terminaram o ensino primário, básico e secundário. A dispersão das distribuições, medida pela variância, mantém-se relativamente constante, independentemente do nível educacional. A forma das funções densidade, por outro lado, vai-se alterando à medida que consideramos indivíduos com nível de escolaridade mais elevado, nomeadamente no que concerne à massa localizada nos extremos das distribuições.

Esta situação, que à partida pode parecer contraintuitiva, já que na literatura tem sido sistematicamente encontrada evidência de uma relação positiva entre o estado de saúde mental e o nível educacional dos indivíduos (OECD, 2012), pode ter uma explicação simples. Considera-se a hipótese seguinte: as expectativas de quem termina um curso superior em termos de tempo necessário para encontrar um emprego, tipo de funções a desempenhar ou nível salarial a auferir, serão certamente superiores às de indivíduos que não possuem estudos, ou que não possuem um curso superior. Tendo em conta a atual situação econó- mica e financeira que Portugal atravessa, bem como o facto de a simples posse de um grau de ensino superior já não ser um fator diferenciador no mercado de trabalho, principalmente entre as gerações mais jovens, é natural que indivíduos que tenham completado o ensino superior também enfrentem dificuldades relacionadas com a procura de emprego. Estes indivíduos vêmse assim confrontados com uma realidade para a qual não estavam preparados, sendo que esta situação afeta a sua saúde mental de forma mais negativa do que ocorre em indivíduos com menor nível de escolaridade e, consequentemente, expectativas menos ambiciosas no que toca à sua situação no mercado de trabalho.

Focando a análise especificamente no grupo de indivíduos pertencentes ao escalão etário entre os 18 e os 35 anos de idade que se encontram em situação de desemprego, a figura 11 apresenta as funções densidade das distribuições da escala de saúde mental e vitalidade por nível de escolaridade.

O grupo de indivíduos até 35 anos, sem qualquer grau de escolaridade completo, que se encontravam desempregados à data de preenchimento do questionário tem um número reduzido de elementos (apenas 3), o que põe em causa a robustez dos resultados. No entanto, para os restantes níveis de escolaridade, cujo número de observações varia entre 15 e 20, verifica-se uma diminuição do valor médio da escala de saúde mental e vitalidade com o nível de educação. Enquanto os jovens desempregados com ensino básico completo têm uma escala média de 60, este valor reduz-se para 54,9 entre os que completaram o ensino básico e subsequentemente para 51,4 para os que possuem uma educação a nível universitário. Atentando a forma das distribuições apresentadas na figura 11, verifica-se que esta situação deriva de, por um lado, um au- 
mento da massa da distribuição nas pontuações mais baixas e, por outro lado, uma diminuição nas pontuações mais elevadas.

Ao realizar esta análise para as restantes faixas etárias já não se obtém este padrão de forma tão marcada (resultados não apresentados), o que leva a crer que a dinâmica existente na faixa etária até aos 35 anos é distinta da das restantes.

Alguns pontos devem, contudo, ser alvo de uma discussão mais aprofundada. O primeiro deles é a dimensão da amostra. De facto, as conclusões re-
SEM ESCOLARIDADE

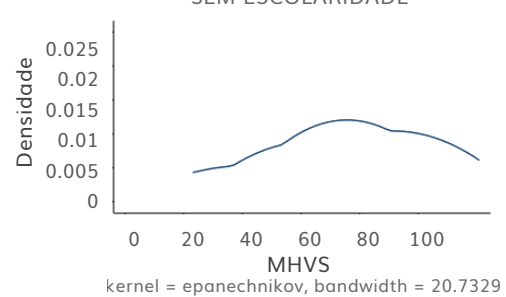

ENSINO SECUNDÁRIO

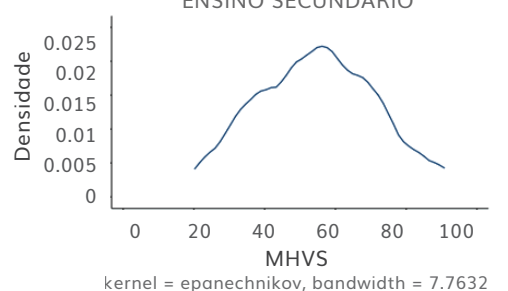

ENSINO BÁSICO

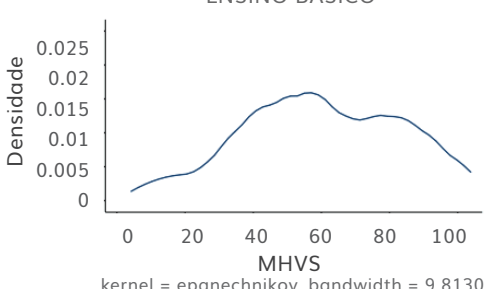

ENSINO UNIVERSITÁRIO

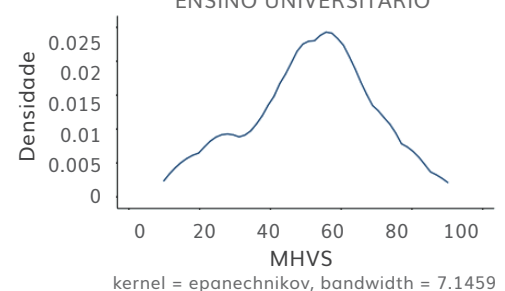

Figura 11. Distribuição MHVS por nível de escolaridade

Fonte: elaboração própria a partir dos questionários realizados nos municípios da Amadora, Lisboa, Mafra e Oeiras, em 2014 e 2015

tiradas da análise das figuras acima apresentadas baseiam-se num reduzido número de observações, pelo que podem não apresentar robustez estatística. Adicionalmente, importa relembrar que a escala de saúde mental e vitalidade que temos vindo a utilizar ao longo desta análise não é um indicador de natureza científica. Pelo contrário, é construído com base em informação auto reportada pelos indivíduos. Não só os indivíduos percecionarão a sua saúde mental de forma distinta, como também possivelmente as suas respostas terão em conta estados de saúde mental passados. Outra hipótese que podemos conjeturar prende-se com o facto de indivíduos com maior nível de educação conseguirem avaliar o próprio estado de saúde mental com maior precisão.

\subsection{Análise por concelho}

Uma vez que o inquérito foi realizado em quatro concelhos distintos da Área Metropolitana de Lisboa, uma análise natural dos dados consiste em avaliar a existência de eventuais diferenças entre concelhos. A análise seguirá uma lógica semelhante à realizada na subsecção anterior, embora com menor exaustividade.

Das 1.609 observações que compõem a amostra utilizada, $444(27,6 \%)$ correspondem ao concelho da Amadora e 373 (23,2\%) ao de Lisboa. As observações referentes aos concelhos de Mafra e Oeiras ascendem a $339(21,1 \%)$ e 453 $(28,2 \%)$, respetivamente.

Estes concelhos apresentam algumas disparidades entre si, nomeadamente no que toca ao grau de escolaridade das populações residentes. De facto, os concelhos de Lisboa e Oeiras possuem uma percentagem da população com ensino superior bastante acima da verificada nos restantes concelhos. Uma vez que a evidência existente na literatura aponta para uma relação positiva entre o nível de escolaridade e o estado de saúde mental (OECD, 2012), estas diferenças educacionais podem traduzir-se em diferenças em termos da escala de saúde mental e vitalidade.

Ao analisar os valores médios da distribuição da MHVS, não se verificam diferenças relevantes entre os quatro concelhos, sendo que a pontuação média de saúde mental varia entre 56,3 na Amadora e os 60,1 em Mafra. A dispersão também se mantém relativamente constante entre concelhos, variando entre os 18,7 (Lisboa) e os 20,8 (Mafra).

O histograma e função densidade da escala de saúde mental e vitalidade por concelho são apresentados na figura 13 . No que concerne aos extremos das distribuições, também não parecem existir diferenças significativas entre concelhos. 

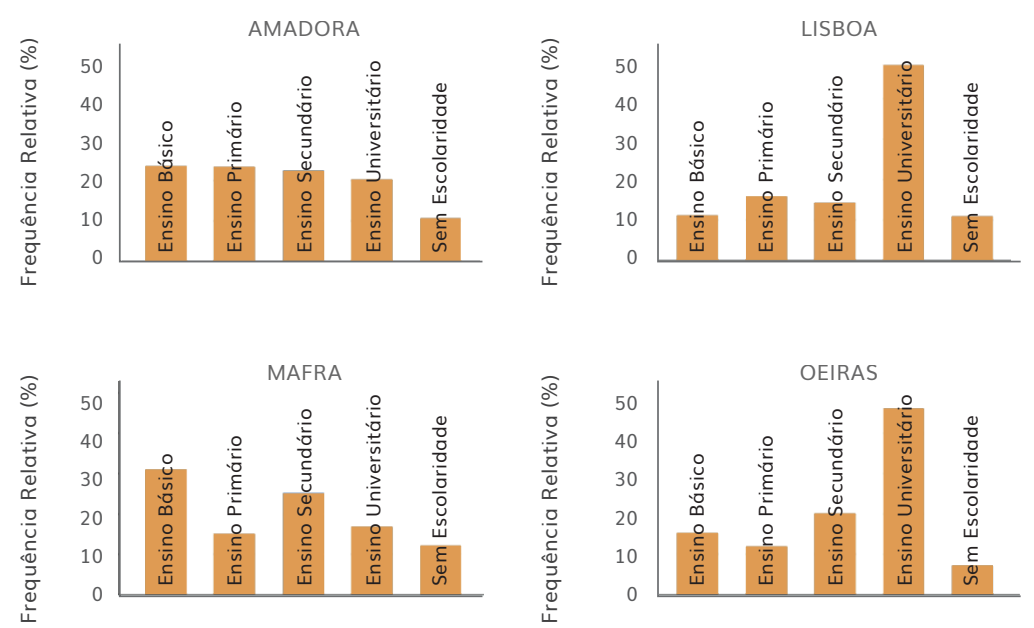

Figura 12. Nível de escolaridade por concelho

Fonte: elaboração própria a partir dos questionários realizados nos municípios da Amadora, Lisboa, Mafra e Oeiras, em 2014 e 2015
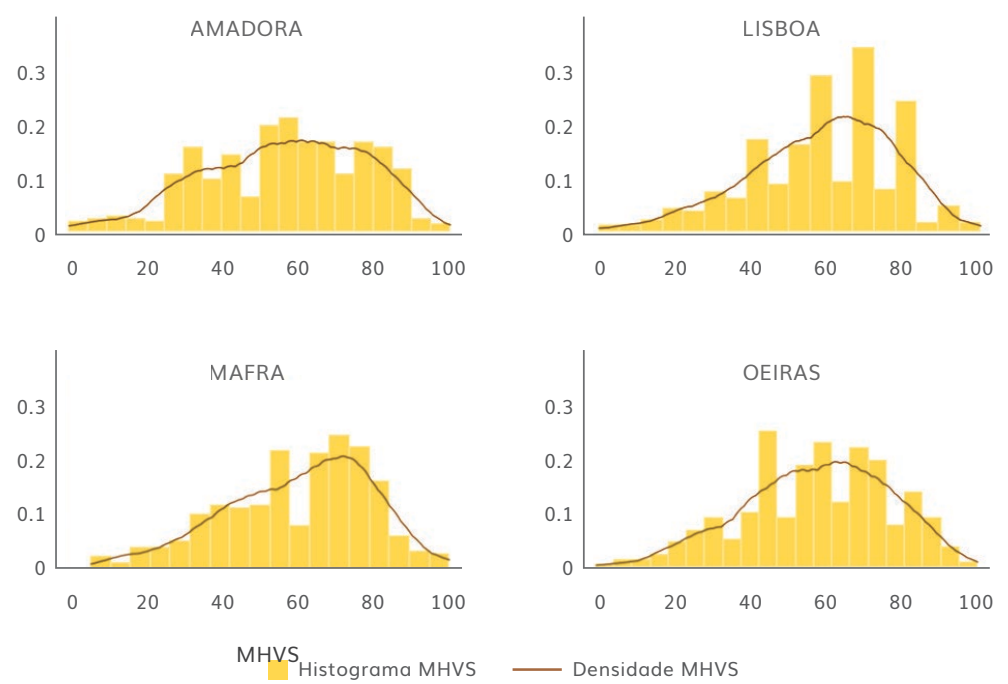

Figura 13. Histograma e função densidade MHVS por concelho

Fonte: elaboração própria a partir dos questionários realizados nos municípios da Amadora, Lisboa, Mafra e Oeiras, em 2014 e 2015
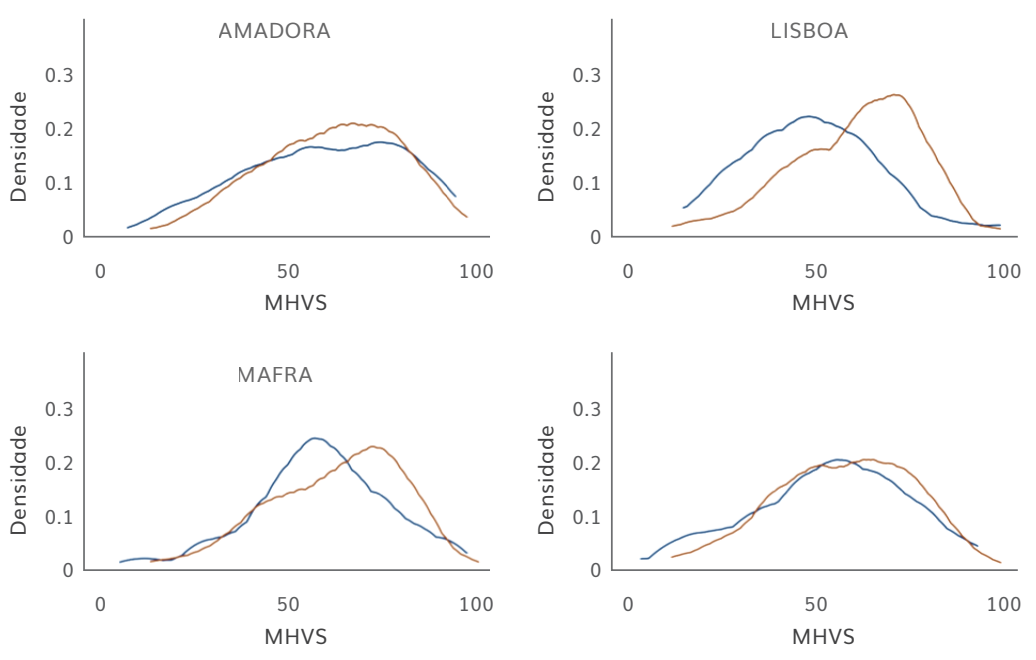

Figura 14. Distribuição MHVS por concelho

Fonte: elaboração própria a partir dos questionários realizados nos municípios da Amadora, Lisboa, Mafra e Oeiras, em 2014 e 2015
Uma análise à função densidade da MHVS, por concelho, comparando a população empregada com a desempregada (figura 14) já aponta para a existência de padrões distintos.

Enquanto nos concelhos da Amadora e Oeiras a função densidade da MHVS é sensivelmente a mesma para indivíduos empregados e desempregados, tal não ocorre nos casos de Lisboa e Mafra. Efetivamente, no concelho de Lisboa a função densidade obtida para os desempregados situa-se à direita da dos empregados, independentemente da escala de saúde mental e vitalidade. Já no caso do concelho de Mafra, a função densidade entre indivíduos desempregados tem o seu pico num valor inferior à dos empregados.

Este tipo de situação pode deverse, por exemplo, à existência de programas e iniciativas bem sucedidas a nível municipal, que contribuam para combater o isolamento e exclusão social dos indivíduos desempregados e simultaneamente promover a sua integração no mercado de trabalho e na sociedade.

\section{Conclusão}

O contributo deste capítulo encontra-se na análise do impacto do desemprego jovem na perceção da saúde mental usando como ferramenta principal a estatística descritiva, em que os dados são analisados de uma forma mais detalhada e em toda a sua extensão.

Esta análise assentou sobretudo na escala de saúde mental e vitalidade (MHVS) a qual foi analisada com base nas variáveis consideradas como fatores de risco para a saúde 
mental, nomeadamente, idade (faixas etárias), género, nível de escolaridade e situação perante o mercado de trabalho.

Da análise da amostra, sem distinguir entre empregados e desempregados, é possível concluir que a generalidade dos indivíduos tem uma boa saúde mental uma vez que mais de $50 \%$ dos indivíduos inquiridos obtêm uma pontuação (na escala de saúde mental e vitalidade) superior a 50. Quando o foco da análise passa a ser os indivíduos desempregados, o padrão é diferente. Os jovens, indivíduos com menos de 35 anos, e com um nível de escolaridade mais elevado, são os que reportam pior saúde mental. Conjeturamos que este facto advenha das elevadas expectativas que estes jovens têm face às oportunidades de emprego, as quais não são satisfeitas pelo mercado de trabalho.

Ao nível do concelho, em Lisboa e Mafra os indivíduos desempregados têm uma escala de saúde mental e vitalidade ligeiramente abaixo dos indivíduos empregados. Já nos concelhos da Amadora e Oeiras não há evidência de uma diferença entre a saúde mental auto reportada pelos indivíduos empregados e desempregados. Uma possível explicação para este facto pode assentar nas políticas ao nível do município que contribuam para combater o isolamento e exclusão social dos indivíduos desempregados e simultaneamente promover a sua integração no mercado de trabalho e na sociedade. De facto, os municípios de Oeiras e da Amadora têm adotado políticas municipais específicas neste sentido, entre as quais se incluem a criação de estruturas de apoio personalizado com vista à resolução de problemas profissionais e de formação. Existem também incentivos à criação de emprego, tanto a nível de isenções fiscais para pequenas e médias empresas, como apoios financeiros para novos projetos empresariais apresentados por desempregados locais. De realçar o facto de algumas destas medidas terem sido implementadas já em 2007, o que pode indicar a existência de vantagens em atuar cedo.

Em suma, podemos afirmar que são os jovens com menos de 35 anos e com maior grau de escolaridade o grupo mais vulnerável. Sugerimos, desta forma, que as políticas que visam combater o desemprego deverão, não só, ter um foco especial no desemprego jovem como estar aliadas a políticas que visem promover a saúde mental.

\section{Agradecimentos}

Este estudo foi desenvolvido no âmbito do projeto de investigação PTDC/ATP-GEO/4101/2012, SMAILE, Saúde Mental - Avaliação do Impacto das Condicionantes Locais e Económicas, financiado por Fundos FEDER através do Programa Operacional Factores de Competitividade - COMPETE e por Fundos Nacionais através da FCT Fundação para a Ciência e a Tecnologia. Agradece-se ao grupo de investigação do projeto SMAILE (Adriana Loureiro, Benedetto Saraceno, Carla Nunes, Graça Cardoso, Joana Lima, José Caldas de Almeida, João Ferrão, Manuela Silva, Maria do Rosário Partidário e Paula Santana) pelos contributos ao longo destes dois anos de desenvolvimento do projeto.

Agradece-se também a Helena Peixoto e Cristina Nunes por todo o suporte prestado na realização dos questionários e à equipa de entrevistadores, Adriana Loureiro, Ângela Freitas, Catarina Barros, Cláudia Costa, Joana Lima, Maria Lucília Cardoso, Paula Santana, Ricardo Almendra e Tiago Cruz.

Um agradecimento às Câmaras Municipais da Amadora, Lisboa, Mafra e Oeiras, Juntas de Freguesia e outras instituições destes concelhos por terem aceite participar no estudo SMAILE, colaborando e apoiando a realização dos questionários à população residente. Agradece-se especialmente aos respetivos presidentes, vereadores e técnicos das câmaras municipais referidas acima (Carla Tavares, Cristina Farinha e Ana Moreno (Câmara Municipal da Amadora); Fernando Medina, João Afonso e Teresa Craveiro (Câmara Municipal de Lisboa); Hélder Silva e Aldevina Rodrigues (Câmara Municipal de Mafra); e Paulo Vistas, Marlene Rodrigues e Marta Camilo (Câmara Municipal de Oeiras)).

\section{Referências Bibliográficas}

Artazcoz, L., Benach, J., Borrell, C., \& Cortes, I. (2004). Unemployment and mental health: understanding the interactions among gender, family roles and social class. American Journal of Public Health, 94(1), 82-88.

Backhans, M., \& Hemmingson, T. (2012). Unemployment and mental health - Who is (not) affected?. European Journal of Public Health, 22(3),429-433. doi: http://dx. doi. org/10.1093/eurpub/ckr059 
Banerji, A., Saksonovs, S., Lin, H., \& Blavy, R. (2014). Youth Unemployment in Advanced Economies in Europe: Searching for solutions.

Breslin, M., \& Breslin, T. (2013). Unemployment and psychological well-being in post celtic tiger Ireland. Irish Journal of Psychology, 34(2), 67-80. doi:10.1080/030339 10.2013.796584

Caldas de Almeida, J., \& Xavier, M. (2013). Estudo Epidemiológico Nacional de Saúde Mental: $1^{\circ}$ Relatório. Lisbon: Nova Medical School, Faculdade de Ciências Médicas.

Clemens, M., Popham, F., \& Boyle, P. (2015). What is the effect of unemployment on all-cause mortaliy? A cohort study using propensity score matching. European Journal of Public Health, 25(1), 115-121.

Dancey, C., \& Reidy, J. (2004). Statistics Without Maths for Psychology: Using SPSS for Windows. London: Prentice Hall.

Dukerich, J. (2001). Role transitions in organizational life: An identity-based perspective by Blake E. Ashforth. The Academy of Management Review, 26(4), 670-672.

European Commission (2015). Addressing youth unemployment in the EU.

Ferreira, L. (2000). Criação da versão Portuguesa do MOS SF-36, Parte I - Adaptação Cultural e Linguística. Acta Médica Portuguesa, 13, 55-66.

International Labour Organisation (2015). Global Employment trends for youth: Scaling up investments in decent jobs for youth. Geneva: ILO.
INE (2015). Desemprego Jovem. Retrieved October 6, 2015, from http://smi.ine.pt/

OECD (2012). Sick on the job? Myths and Realities about Mental Health and Work. OECD Publishing.

OECD (2014). Making Mental Health Count: The Social and Economic Costs of Neglecting Mental Health Care.

OECD (2015). Mental health and older adults. Fact Sheet No. 381.

Paul, K., \& Moser, K. (2009). Unemployment impairs mental health: Meta-analyses. Journal of Vocational Behaviour, 74(3), 264-282. doi:10.1016/j.jvb.2009.01.001

Syme, S. (1996). Rethinking disease: Where do we go from here?. Annals Epidemiology, 6(5), 463-468.

Ware, J., Snow, K., Kosinski, M., \& Gandek, B. (1993). SF36 Health Survey: manual and interpretation guide. Boston: The Health Institute, New England Medical Center.

World Health Organisation (2007). Mental Health: Strenghtening mental health promotion. World Health Organisation. Fact Sheet No. 220.

World Health Organisation (2011). Impact of economic crisis on mental health. 